\title{
The use of bacteriophages for P. aeruginosa biofilm control
}

\author{
D. P. Pires, S. Sillankorva, J. Azeredo \\ Department of Biological Engineering \\ University of Minho \\ Braga, Portugal \\ jazeredo@deb.uminho.pt
}

\begin{abstract}
Pseudomonas aeruginosa is a relevant opportunistic pathogen frequently associated with several nosocomial infections and, worryingly, this bacterium shows a low antibiotic susceptibility. One of its virulence factors is related with the ability to adhere to surfaces and also human epithelium and form virulent biofilms. This work describes the isolation and characterization of lytic phages capable to infect antibiotic resistant $P$. aeruginosa strains. It is also described herein the potential of the new isolated phages for planktonic cells and biofilm control. According to the results of this work, the isolated phages showed different spectra of activity and most of them were efficient even against $P$. aeruginosa multidrug resistant strains. Furthermore, the biofilm infection assays revealed that phages can be a good strategy to combat virulent biofilms achieving significant reductions in the number of biofilm cells.
\end{abstract}

Keywords: Bacteriophages, P. aeruginosa, Biofilms, Control

\section{CONTEXT}

Pseudomonas aeruginosa is an ubiquitous organism which has emerged as a major threat in the hospital environment. This bacterium is the most frequently isolated Gram-negative organism in bloodstream and wound infections, pneumonia, intra-abdominal and urogenital sepsis and is a serious problem infecting immunocomprimised patients with cystic fibrosis (CF), severe burns, cancer, AIDS, etc. [1-2]. One of the most worrying characteristics of this bacterium is its low antibiotic susceptibility which can be attributed to a concerted action of multidrug efflux pumps with chromosomally-encoded antibiotic resistance genes and the low permeability of the bacterial cellular envelopes [3]. The overuse of antibiotics has also significantly increased the emergence of antimicrobial multi-resistant bacteria and consequently, the treatment of most chronical $P$. aeruginosa infections with antibiotics is notoriously difficult [3-4]. Additionally, P. aeruginosa has an inapt ability to adhere to surfaces and form virulent biofilms which are particularly difficult to eradicate [5-6]. Biofilm formation is an important bacterial survival strategy and, in humans, biofilms are responsible for numerous pathologies usually associated with the use of medical devices [7-8]. In this way, new alternative strategies to antibiotherapy are highly desired by the worldwide medical and scientific community

\footnotetext{
$1^{\text {st }}$ Portuguese Meeting in Bioengineering, February 2011

Portuguese chapter of IEEE EMBS

Instituto Superior Técnico, Technical University of Lisbon
}

and bacteriophages (phages) appear as one attractive solution for this problem [9-11].

Phage therapy is based on the use of lytic phages to combat bacterial infections, including multi-drug resistant bacteria, and has many advantages comparatively to antibiotics: they are very specific and efficient for their target bacteria which reduces the destruction of the natural flora on the host; they are not pathogenic for man; and they need their target bacteria to grow and only persist as long as the bacteria they are targeting is present $[9,12]$.

\section{GOALS}

The aim of this work was the isolation and characterization of novel $P$. aeruginosa phages and also the evaluation of their efficiency on planktonic cultures and mono-species biofilms.

\section{IMPLEMENTATION}

\section{A. Isolation and characterization of phages}

Bacteriophages were isolated from 2 hospital effluents provided by Hospital de São João (Porto, Portugal). These effluents were enriched with $4 P$. aeruginosa strains (ATCC 10145, CECT 111, PAO 1 and a clinical isolate (CLIN)) in $2 \times$ Tryptic Soy Broth (TSB) medium. This solution was incubated $\left(37^{\circ} \mathrm{C}, 120 \mathrm{rpm}\right)$ during 48 hours and then centrifuged to collect the supernatant for the spot tests (Fig. 1) indicative of the existence of phage(s). Each inhibition halo was further purified with toothpicks and papers, to isolate all different phages.

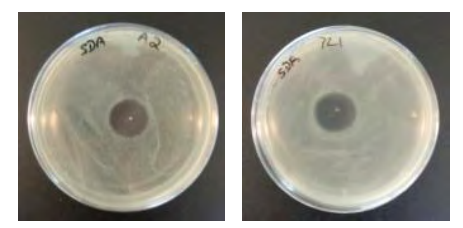

Figure 1. Examples of a spot test of enriched effleunt on lawns of P. aeruginosa ATCC 10145 and PAO 1.

Overall, 17 phages with different characteristics were isolated and 4 of them were selected to perform planktonic 
cells infection assays. This selection was done according to the results of the lytic spectra. To evaluate the lytic spectra of all isolated phages, each phage was tested against 35 different $P$. aeruginosa strains. 31 of these strains were clinical isolates provided by Hospital de São Marcos (Braga, Portugal). To perform the lytic spectra assay, one drop of each serial diluted phage suspension was placed into the different bacterial lawns and incubated overnight at $37^{\circ} \mathrm{C}$. In the following day, the susceptibility of each host to the different phages was evaluated.

SDS-PAGE experiment was also performed in order to analyze the structural proteins of the 4 selected phages. After the preparation of the gels, the samples were added and the gels were run at $100 \mathrm{~V}(80 \mathrm{~mA})$. After electrophoresis, the gels were stained with silver nitrate.

\section{B. Phage infection}

\section{Infection of planktonic cells}

The infection of planktonic cells was done in two different stages of bacterial growth - exponential and stationary phases. To perform the planktonic cell infections at the stationary stage, the 4 hosts were inoculated overnight in TSB medium at $37^{\circ} \mathrm{C}(120 \mathrm{rpm})$. The resultant cellular suspensions were diluted with TSB medium in order to have an optical density $\left(\mathrm{OD}_{600 \mathrm{~nm}}\right)$ approximately 0.75 . To do the planktonic cells infection at exponential stage, an overnight pre-inoculum was used to inoculate fresh TSB medium which were allowed to grow until achieving an optical density $\left(\mathrm{OD}_{600 \mathrm{~nm}}\right)$ of 0.5 . In all experiments, phages were added to the respective hosts in order to obtain a multiplicity of infection (MOI) of 1 . The control experiments were performed with SM Buffer instead of phages. The infection of planktonic cell assays were performed in 96 -well microtiter plates $\left(37^{\circ} \mathrm{C}, 120 \mathrm{rpm}\right)$ and the optical density $\left(\mathrm{OD}_{600 \mathrm{~nm}}\right)$ was regularly read.

\section{Characterization of $P$. aeruginosa biofilms}

Based on the phage infection experiments of the planktonic cells, only $P$. aeruginosa ATCC 10145 and PAO 1 strains were used in biofilm assays. Biofilm formation was carried out in 24-well microplates containing $1 \mathrm{ml}$ of Yeast Peptone Dextrose (YPD - $10 \mathrm{~g} \mathrm{l}^{-1}$ Yeast Extract, $20 \mathrm{~g} \mathrm{l}^{-1}$ Peptone, $20 \mathrm{~g} \mathrm{l}^{-}$ ${ }^{1}$ Dextrose) medium and $10 \mu 1$ of cellular suspension with an optical density $\left(\mathrm{OD}_{600 \mathrm{~nm}}\right)$ of 1.0. Biofilms were formed during 24 hours with medium renewal every 12 hours and the plates were incubated at $37^{\circ} \mathrm{C}$ in an orbital shaker $(120 \mathrm{rpm})$. The amounts of bacteria attached to the biofilms were determined by CFU counts using microdrop technique. Briefly, the wells of microplates were washed with saline solution $(0.9 \% \mathrm{NaCl})$ to remove unattached bacteria and then $1 \mathrm{ml}$ of fresh saline solution was added to each well and the biofilm scraped with a cell scrapper prior to sonication $(5 \mathrm{~min})$. After this, the cellular suspension of each well was removed, centrifuged $(5 \mathrm{~min}$, $9,000 \times \mathrm{g}, 4^{\circ} \mathrm{C}$ ) and the pellet resuspended in $1 \mathrm{ml}$ of saline solution $(0.9 \%)$. The samples were diluted in saline solution $(0.9 \%)$ and one drop $(10 \mu \mathrm{l})$ was placed into a Petri plate containing YPD solid medium and allowed to run down the plate. The plates were incubated at $37^{\circ} \mathrm{C}$ during 16 to $18 \mathrm{~h}$ and after the CFU's were counted.

\section{Biofilm infection}

The biofilm infection was done with two different phages phiIBB-PAA2 and phiIBB-PAP21. After 24 hours of biofilm formation, all the medium and planktonic bacteria were removed from each well and washed with fresh YPD medium. Following that, $500 \mu \mathrm{l}$ of fresh YPD medium and $500 \mu \mathrm{l}$ of phage solution or SM Buffer, in case of control experiments, were added. The multiplicity of infection (MOI) used for the experiments was 1 . The plates were incubated in an orbital shaker $(120 \mathrm{rpm})$ at $37^{\circ} \mathrm{C}$ and samples were taken after 2,6 and 24 hours of infection for CFU and PFU counts. The CFU counts were determined as previously described by microdrop technique and for PFU counting the Small Drop method described by Mazzoco et al. (2008) was used [13].

\section{RESUltS}

\section{A. Phage characterization}

In the present study, 17 new phages for $P$. aeruginosa were isolated from hospital effluents using three collection strains and a clinical strain which were tested for efficiency against 35 clinical strains. Most phages showed lytic activity against several clinical isolates, even against multi-resistant strains. The least efficient isolated phages were those with the clinical $P$. aeruginosa isolate as host where the percentages of strains infected varied between 17 and 54 percent, while the most efficient phages, the ones with CECT 111 as propagation strain, were capable of lysing approximately 60 to 89 percent of the clinical strains tested (data not shown).

After lytic spectrum assays, one phage for each host was selected for further characterization and also for use in planktonic and biofilm infection experiments. The criteria used for this selection was the number of strains that each phage was able to infect. Accordingly, the selected phages were: phiIBB-PAA2, phiIBB-PAC23, phiIBB-PACL12 and phiIBBPAP21, respectively. Phages phiIBB-PAA2 and philBBPAP21 were analysed by Transmission Electron Microscopy and were found to belong to the "T7-like" genus of the Podoviridae family of phages (Fig. 2A). Phage structural proteins, analyzed by SDS-PAGE (Fig. 2B), showed that both phages phiIBB-PAC23 and phiIBB-PAP21 have a high degree of homology and thus belong also to the "T7-like" genus.

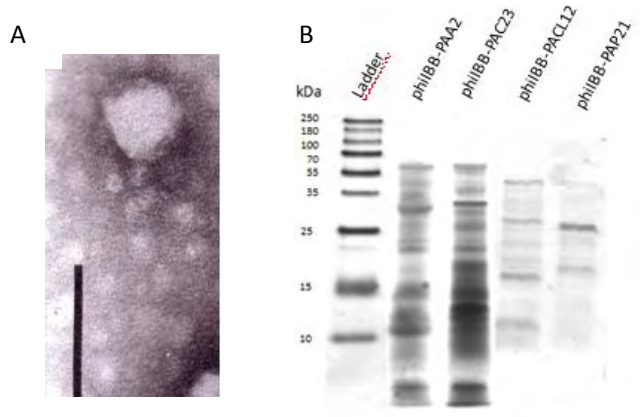

Figure 2. Characterization of phages A) Phage phiIBB-PAA2 observed by Transmission Electron Microscopy (TEM) (bar corresponds to $100 \mathrm{~nm}$ ); B) SDS-page of structural proteins of $P$. aeruginosa phages philBB-PAA2, phiIBB-PAC23, phiIBB-PACL12 and phiIBB-PAP21 


\section{B. Infection of planktonic cells}

After phage selection and characterization, phage infection experiments were performed in exponential growing and stationary cells (Fig. 3). There was a clear difference in the infection patterns after infection of cells at the stationary and exponential phases with the 4 different phages. Phage philBBPAA2 appeared to have a greater capacity of infecting $P$. aeruginosa host, followed by phage phiIBB-PAP21. Phage phiIBB-PACL12, although having a broad spectrum of activity in infecting of different isolates, did not cause a reduction of the optical density of neither exponentially growing nor stationary phase cells. This suggests that although phages can be selected based on their broad activity, this doesn't necessarily guarantee their effectiveness in controlling their respective host population. Nevertheless, two of the used phages, phages phiIBB-PAA2 and phiIBB-PAP21, were both efficient towards exponential as well as stationary phase cells and caused a reduction over $50 \%$ of the initial optical density $\left(\mathrm{OD}_{600 \mathrm{~nm}}\right)$.
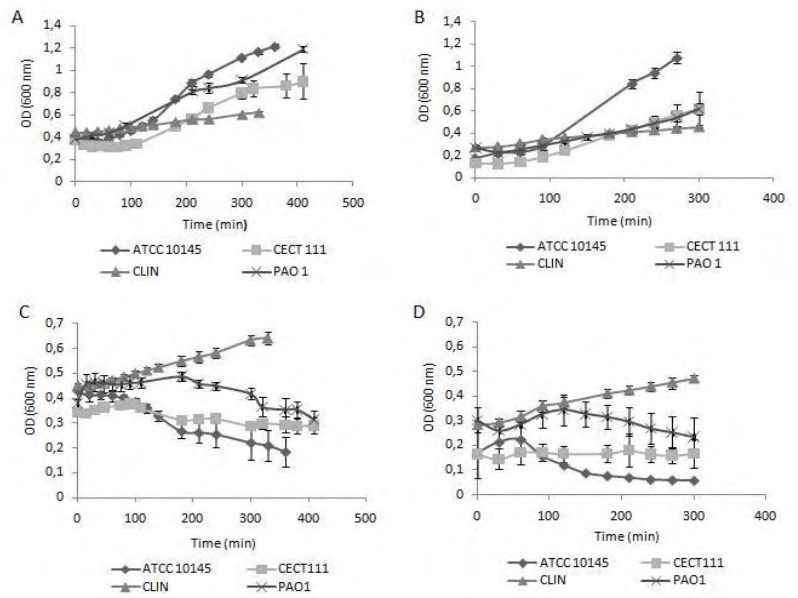

Figure 3. Infection of planktonic $P$. aeruginosa cultures with phages philBB-PAA2 (ATCC 10145), phiIBB-PAC23 (CECT 111), phiIBB-PACL12 (CLIN) and phiIBB-PAP21 (PAO 1). A) Control experiments with stationary phase cultures; B) Control experiments with exponentially growing cells; C) Phage infection experiments with stationary cultures; D) Phage infection experiments with exponentially growing cultures.

\section{Biofilm infection}

It is expected that the phage infection in planktonic cells would be more efficient than in biofilms due to the biofilm architecture which difficult the access of phage into bacteria [14]. Due to the inefficient activity of phages phiIBB-PACL12 and phiIBB-PAC23 against planktonic cultures, only two phages, philBB-PAA2 and phiIBB-PAP21, were chosen for $P$. aeruginosa biofilm infection assays.

Before $P$. aeruginosa biofilm infection, the number of CFU counts of 24 hours biofilms of both strains chosen for this experiment were determined (Fig. 4) and presented similar results.

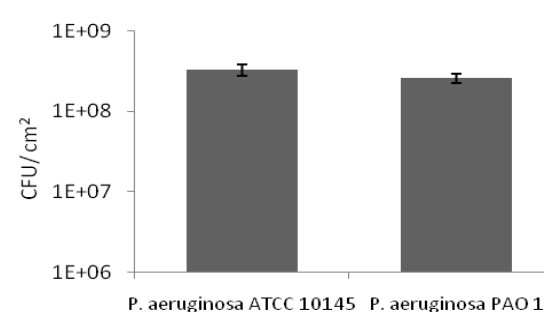

Figure 4. Enumeration of the number of viable cells present in P. aeruginosa ATCC and PAO 1 biofilms formed during 24 hours

As previously described, the phage infection assays of $P$. aeruginosa ATCC 10145 and PAO1 were performed in 24 hours old biofilms (Fig. 5).

Both phages, tested against 24 hours old biofilms, caused a significant reduction on the biofilm cells already after 2 hours of infection and the reduction was further enhanced after 6 hours of biofilm treatment reaching a reduction of almost $3 \mathrm{log}$ with phage phiIBB-PAP21 and a $2 \log$ reduction with phage phiIBB-PAA2 (Fig. 5A).

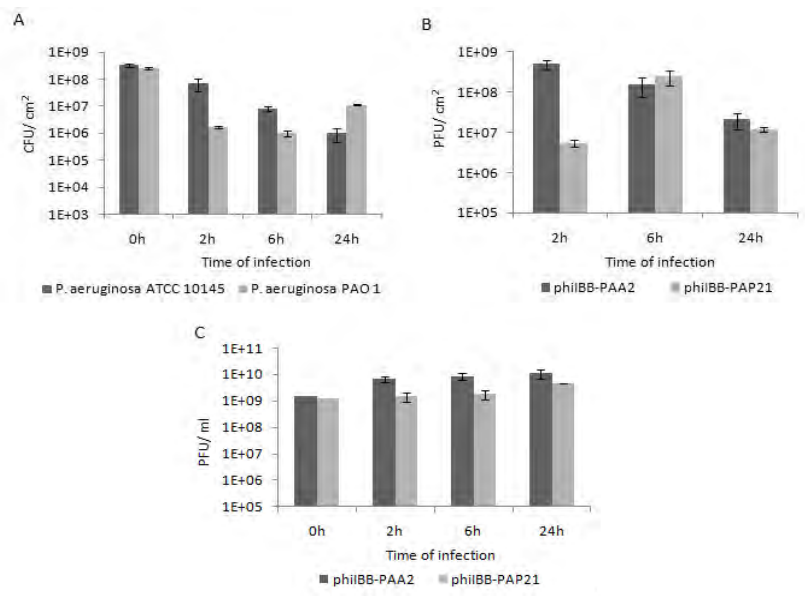

Figure 5. Infection of $P$. aeruginosa ATCC 10145 and PAO1 biofilms with phages phiIBB-PAA2 and phiIBB-PAP21, respectively. A) Number of viable cells before and after infection; B) Number of phages (PFU) attached to the biofilms; C) Number of phages (PFU) released from the infected biofilms.

Despite the lower activity against planktonic cells, phage phiIBB-PAP21 caused a higher reduction of viable cells from the $P$. aeruginosa PAO1 biofilms after 2 and 6 hours of treatment than phage phiIBB-PAA2 in $P$. aeruginosa ATCC 10145 biofilms. Similar reductions of viable cells have been observed for $P$. fluorescens phages [15] and there is only one work in literature where complete eradication of 24 hours Enterobacter cloace biofilms was achieved by a combination of 3 different phages [16]. The main difference between the two phages used in this work for $P$. aeruginosa biofilm control experiments concerns the resistance of the bacteria to the phages. The interaction of phages and bacteria during long periods of time can result in the emergence of phage resistant bacteria [17-18]. Biofilm cells of $P$. aeruginosa PAO1, the host of phage phiIBB-PAP21, acquired resistance to the phage resulting in an increase on the amount of cells after 24 hours of biofilm treatment (Fig. 5A). On the other hand, phage philBBPAA2 continued to destroy biofilm cells of $P$. aeruginosa 
ATCC 10145 and there were no evidences of cells becoming resistant, even after 24 hours of phage infection.

One unexpected result obtained was in terms of the number of phages attached to the biofilms (and the surface of the well) measured by plaque forming units (PFU) counts (Fig. 5B). It appears that initially phage phiIBB-PAA2 adsorbed to the biofilms, however after cell lysis the number of phages present in the biofilms decreased by almost 2 logs. Unlike with phage phiIBB-PAA2, the amount of phiIBBPAP21 phages continued to increase until 6 hours of infection, however, as in phiIBB-PAA2 experiments, there was again observed a significant decrease of the number of phages attached after 24 hours of biofilm cell infection.

Overall, this work revealed the efficacy of phages in cell lysis even against multidrug resistant bacteria. Furthermore, both novel isolated phages were well capable of controlling $P$. aeruginosa biofilms.

\section{CONCLUSIONS}

In this study it was possible to conclude that most of the isolated phages have a great lytic capacity against $P$. aeruginosa clinical strains achieving high percentages of lysis.

This study also revealed that phages may be a good alternative to traditional antimicrobials for the control of $P$. aeruginosa biofilms; however, short periods of treatment seem to be a better solution avoiding the emergence of phage resistant hosts.

\section{PlanNED DEVELOPMENTS}

Some alternative approaches can be very interesting for the future work. The combination of phages with chemical agents such as detergents, disinfectants and antibiotics can result in a good option for the eradication of $P$. aeruginosa biofilms from different clinical surfaces. Another alternative strategy involves the use of a cocktail with different phages in order to obtain a solution with a greater range of action. Finally, the genetic manipulation of phages may also be an important future approach in order to obtain phages with broad lytic spectra and also with high ability to control resistant phenotypes.

\section{ACKNOWLEDGMENT}

Authors gratefully acknowledge to Hospital de Braga for providing the $P$. aeruginosa clinical strains. The authors are also grateful to Dr. Hans Ackermann for the morphological characterization of the phages.

\section{REFERENCES}

[1] Driscoll, J.A., Brody, S.L. and Kollef, M.H., "The Epidemiology, Pathogenesis and Treatment of Pseudomonas aeruginosa Infections". Drugs, 2007. 67(3): p. 351-368.

[2] Page, M.G.P. and Heim, J., "Prospects for the next anti-Pseudomonas drug". Curr Opin Pharmacol, 2009. 9(5): p. 558-565.

[3] Lambert, P.A., "Mechanisms of antibiotic resistance in Pseudomonas aeruginosa". J R Soc Med, 2002. 95(41): p. 22-26.

[4] Cunha, B.A., "Pseudomonas aeruginosa: resistance and therapy". Semin Respir Infect, 2002. 17(3): p. 231-239.

[5] Drenkard, E., "Antimicrobial resistance of Pseudomonas aeruginosa biofilms". Microbes Infect, 2003. 5(13): p. 1213-1219.

[6] Stewart, P.S. and William Costerton, J., "Antibiotic resistance of bacteria in biofilms". The Lancet, 2001. 358(9276): p. 135-138.

[7] Donlan, R.M., "Biofilms: microbial life on surfaces". Emerg Infect Dis, 2002. 8(9): p. 881-890

[8] O'Toole, G., Kaplan, H. and Kolter, R., "Biofilm Formation as Microbial Development". Annu Rev Microbiol, 2000. 54(1): p. 49-79.

[9] Azeredo, J. and Sutherland, I.W., "The Use of Phages for the Removal of Infectious Biofilms". Curr Pharm Biotechnol, 2008. 9(4): p. 261-266.

[10] Clark, J.R. and March, J.B., "Bacteriophages and biotechnology: vaccines, gene therapy and antibacterials". Trends Biotechnol, 2006. 24(5): p. 212-218

[11] Sulakvelidze, A., "Phage therapy: an attractive option for dealing with antibiotic-resistant bacterial infections". Drug Discov Today, 2005. 10(12): p. 807-809.

[12] Matsuzaki, S., Rashel, M., Sakurai, S., Ujihara, T., Kuroda, M., Ikeuchi, M., et al., "Bacteriophage therapy: a revitalized therapy against bacterial infectious diseases". J Infect Chemother, 2005. 11(5): p. 211 219

[13] Mazzocco, A., Waddell, T.E., Lingohr, E. and Johnson, R. P., "Enumeration of Bacteriophages Using the Small Drop Plaque Assay System", in Bacteriophages: Methods and Protocols, Volume 1: Isolation, Characterization and Interactions. 2008, Humana Press. p. 8185 .

[14] Hanlon, G.W., Denyer, S.P., Olliff, C.J. and Ibrahim, L. J., "Reduction in Exopolysaccharide Viscosity as an Aid to Bacteriophage Penetration through Pseudomonas aeruginosa Biofilms". Appl Environ Microbiol, 2001. 67(6): p. 2746-2753

[15] Sillankorva, S., Oliveira, R., Vieira, M.J., Sutherland, I. and Azeredo, J., "Bacteriophage $\Phi$ S1 Infection of Pseudomonas fluorescens Planktonic Cells versus Biofilms". Biofouling, 2004. 20(3): p. 133 138

[16] Tait, K., Skillman, L.C. and Sutherland, I.W., "The efficacy of bacteriophage as a method of biofilm eradication". Biofouling, 2002. 18(4): p. 305-311.

[17] Bohannan, B.J.M. and Lenski, R.E., "Linking genetic change to community evolution: insights from studies of bacteria and bacteriophage". Ecology Letters, 2000. 3(4): p. 362-377.

[18] Buckling, A. and Rainey, P.B., "Antagonistic coevolution between a bacterium and a bacteriophage". Proc Biol Sci, 2002. 269(1494): p 931-936. 